\title{
Zwischen Profit und medizinischer Erfordernis
}

D ie Fort- und Weiterbildung der Ärzte wird heute fast inflationär diskutiert und bearbeitet. Zweifellos besteht ein kontinuierlicher Bedarf für die Weiterbildung mit universitär geprüftem Wissen, obgleich es aufgrund allseits begrenzter finanzieller Ressourcen immer schwieriger wird, dies auch optimal umzusetzen. Daraus entsteht ein Konflikt zwischen dem ethischen Anspruch des ärztlichen Berufsbildes mit dem Gebot einer leistungsadäquaten Finanzierung und dem profitorientierten Finanzmanagement des deutschen Gesundheitssystems - insbesondere des Klinikbetriebs.

Die moderne Approbationsordnung verschärft die Mängel in der Weiterbildung weiter, da die nicht korrigierten, unzeitgemäß gestalteten Ausbildungs-Curricula die aufgrund der modernen Entwicklung entstandenen Defizite weiterhin nicht abdecken. Fehlende Gesprächskompetenz im Arzt-Patientengespräch, fehlende EDVKenntnisse in einem heute schon fast „papierlosen“ Klinikalltag oder die fehlende Kompetenz, die Seriosität und Qualität der inzwischen kaum zu überblickenden Datenfülle der Forschung sicher zu beurteilen, betreffen jedoch nicht nur die persönliche Qualifikation eines Mediziners, sondern beeinflussen auch die Entwicklung des Krankenhauswesens und die klinische Medizin und damit auch die (pekuniäre) Leistungsfähigkeit einer Klinik!

Neben diesen mehr oder weniger definierten Unzulänglichkeiten, für die zurzeit Weiterbildungsangebote entwickelt und zur Verfügung gestellt werden - zum Beispiel von der Akademie für Wissenschaft, Wirtschaft und Technik an der Universität in Ulm (www.uniulm.de/akademie) -, bestehen grundsätzliche Veränderungen im Gesundheitswesen, die unmittelbar und nachhaltig das Selbstverständnis des ärztlichen Berufes beherrschen. War die Behandlung und - wenn möglich die Heilung der Patienten noch bis vor kurzem primäre Zielvorgabe des Arztberufs, wird dieser Auftrag heute unter den finanziellen Zielen einer profitorientierten Betriebsführung teilweise nachrangig eingestuft. Aufgrund der Technisierung der Medizin beanspruchen medizinische Leistungen jedoch immer höhere finanzielle Ressourcen. Ein erster Paradigmenwechsel bestand darin, dass nur noch solche medizinische Leistungen am Patienten erbracht wurden, für die eine finanzielle $\mathrm{Ab}$ sicherung vereinbart war.

Dieses Verhalten ist durchaus mit dem ethischen Anspruch eines Arztes vereinbar, so lange den Behandlungskatalogen und Leistungsprioritäten ausschließlich medizinische, ärztliche Kriterien zugrunde gelegt werden. Nicht zu akzeptieren ist jedoch, dass Leistungen nur noch unter dem profitorientierten Aspekt erfolgen dürfen. Natürlich setzt die effiziente medizinische Behand- lung von Krankheiten zielorientierte diagnostische Strategien voraus, die im Zweifelsfall teure, unstrittige diagnostische Maßnahmen erfordern. Die Zwänge der modernen Krankenhausfinanzierungssysteme verschärfen das Problem noch weiter, weil sie dazu auffordern, die immer vorhandenen Komplikationsprofile schon im Vorfeld möglichst präzise zu erfassen. Dies erfordert eine sorgfältige, ethisch begründete Vorgehensweise, welche die Verhältnismäßigkeit der eingesetzten Mittel berücksichtigt.

Dieses komplexe, stark veränderte medizinische Umfeld hat einen direkten Einfluss auf die Reputation der Krankenhäuser. Der gute Ruf einer Klinik ist dann in Gefahr, wenn der Umgang mit den Patienten durch die zunehmend spürbaren Defizite der Kommunikationsfähigkeit geprägt ist und durch die ökonomisch begründeten Einschnitte in der medizinischen Leistungsfähigkeit bestimmt wird. Dann ist das Krankenhaus nur noch ein Profitbetrieb, der sich von dem medizinischen Ethos entfernt hat. Daher ist es für die Nachhaltigkeit der zukünftigen Leistungsfähigkeit der Medizin eine unabdingbare Bedingung, die erwähnten Defizite in der Fortund Weiterbildung zu bekämpfen - wofür dann allerdings auch die Umwidmung der Priorität finanzieller Ressourcen eine unausweichliche Begleiterscheinung sein muss.

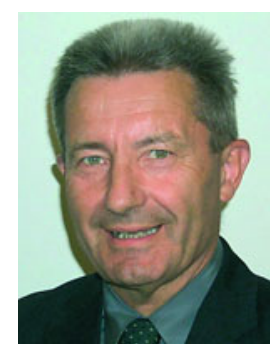

Prof. Dr. Dr. Dr. A. Grünert, Ulm

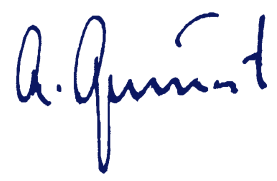

